\title{
A 65-nm CMOS Temperature-Compensated Mobility-Based Frequency Reference for Wireless Sensor Networks
}

\author{
Fabio Sebastiano*, Lucien Breems*, Kofi Makinwa ${ }^{\dagger}$ Salvatore Drago*, Domine Leenaerts* and Bram Nauta ${ }^{\ddagger}$ \\ *NXP Semiconductors, Eindhoven, The Netherlands, Email: fabio.sebastiano@nxp.com \\ ${ }^{\dagger}$ Electronic Instrumentation Laboratory, Delft University of Technology, Delft, The Netherlands \\ ${ }_{\ddagger}^{\ddagger}$ IC Design Group, CTIT Research Institute, University of Twente, Enschede, The Netherlands
}

\begin{abstract}
For the first time, a temperature-compensated CMOS frequency reference based on the electron mobility in a MOS transistor is presented. Over the temperature range from $-55{ }^{\circ} \mathrm{C}$ to $125{ }^{\circ} \mathrm{C}$, its frequency spread is less than $\pm \mathbf{0 . 5 \%}$ after a two-point trim and less than $\pm \mathbf{2 . 7 \%}$ after a one-point trim. These results make it suitable for use in Wireless Sensor Network nodes. Fabricated in a baseline 65-nm CMOS process, the 150 $\mathrm{kHz}$ frequency reference occupies $0.2 \mathrm{~mm}^{2}$ and draws $42.6 \mu \mathrm{A}$ from a 1.2-V supply at room temperature.
\end{abstract}

\section{INTRODUCTION}

Wireless Sensor Networks (WSN) are based on small, cheap and energy efficient nodes. Since the largest fraction of the energy used in each node is spent listening to the channel, synchronous networks are employed to reduce such idle listening time [1]. In that case, the receiver predicts the timeslot that the transmitter will use and turns itself off when no incoming signal is expected. The duty-cycle of the receiver can be lower if the timeslot can be predicted with a smaller error, i.e. if a more accurate time reference is available. Accuracies of a few ppm can be achieved by crystal-controlled oscillators (XCOs), but since such external components should be avoided to reduce the cost and size of the nodes, accuracy must be given up for the sake of integration.

The tradeoff between integration and time/frequency accuracy is also present in the RF front-end. While commercial communication systems require high frequency accuracy, radios for WSN can be optimized to relax such specifications and so frequency accuracies in the order of only a few percent are needed [1] [2]. Thus, it is interesting to investigate which level of accuracy can be reached without external components, with the constraint to operate at the low voltage and power levels typical of WSN supplies.

Recently, much work has been devoted to implementing fully integrated frequency references in standard microelectronic technologies. $L C$ oscillators [3] can provide accuracy and phase noise performances comparable to XCOs; however, their power consumption can hardly be reduced below $100 \mu \mathrm{W}$ due to the limited Q of integrated inductors and the possible need for high-speed frequency dividers. Fully integrated frequency references based on ring oscillators [4] and silicon thermal diffusivity [5] are quite accurate, but dissipate several milliwatts of power. $R C$ oscillators can achieve inaccuracies

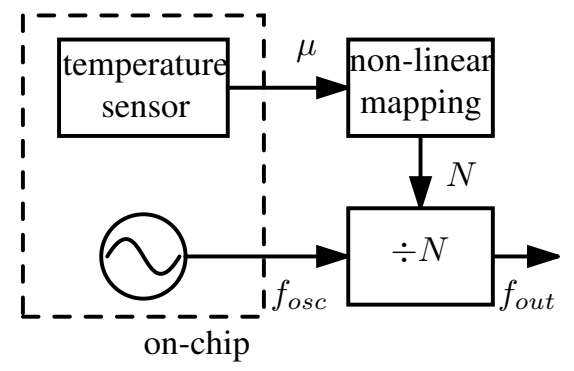

Fig. 1. Block diagram of the frequency reference.

less than $1 \%$ while consuming less than $200 \mu \mathrm{W}$ [6], [7], but their accuracy relies on the availability of on-chip resistors with low or, at least, accurately defined temperature coefficients.

As an alternative, the mobility of charge in a MOS transistor can be employed as a reference. It exhibits low process spread and, although its temperature dependence is large (approximately proportional to $T^{-1.6}$, where $T$ is the absolute temperature), it is well defined for a given process and thus can be compensated for. The effect of process spread can then be removed by a one or two temperature calibration.

In this paper, we explore the level of accuracy that can be achieved by a fully integrated temperature-compensated oscillator that is referenced to electron mobility. The proposed frequency reference comprises a current-controlled relaxation oscillator, in which the current is proportional to the mobility, and a bandgap-based temperature sensor for temperature compensation. Experimental validation of this approach will be provided, demonstrating that, after a two-point calibration, a frequency spread of less than $\pm 0.5 \%$ can be achieved over the military temperature range. The circuit is presented in section II; experimental results are shown in section III and conclusions are drawn in section IV.

\section{Temperature-COMPEnSATED Mobility-BASED REFERENCE}

\section{A. System Architecture}

The proposed frequency reference consists of a mobilityreferenced oscillator, a band-gap temperature sensor (TS) 


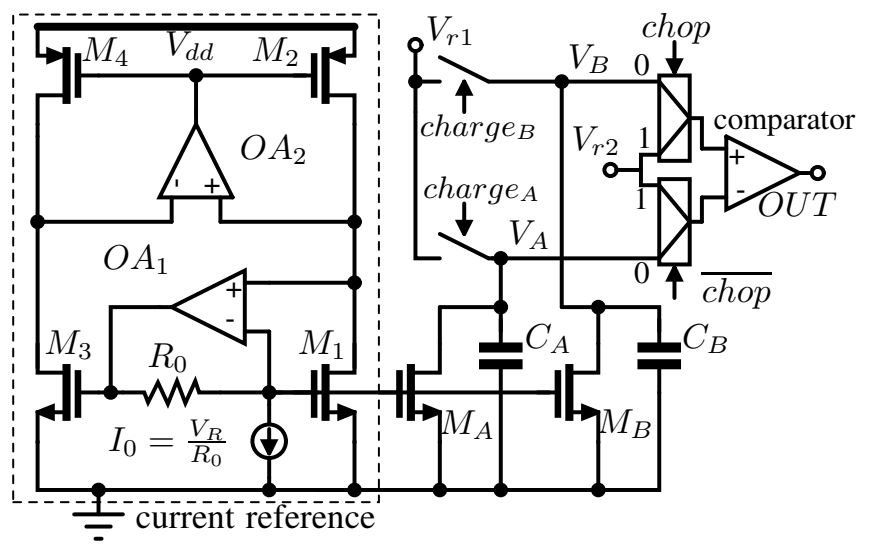

(a)

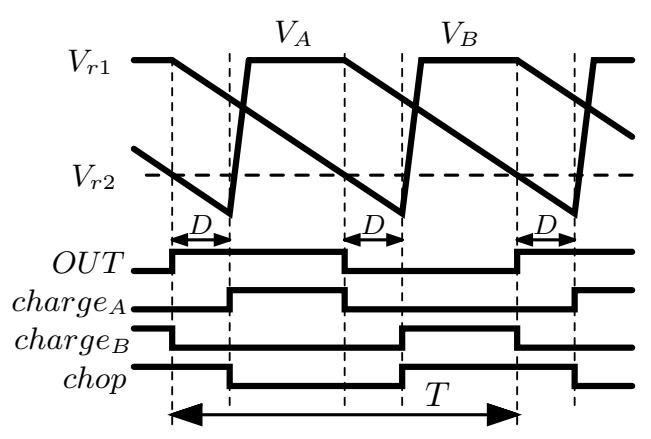

(b)

Fig. 2. Mobility-referenced oscillator (a) and its waveforms (b).

and an external frequency divider (Fig. 1). The mobilityreferenced oscillator generates a frequency $f_{\text {osc }}$ proportional to the electron mobility $\mu_{n}$ in an NMOS transistor. Via a predetermined compensation curve, the digital output of the TS is mapped to a division factor $N$ in such a way that the output frequency $f_{\text {out }}$ remains constant over temperature.

\section{B. Mobility-based oscillator}

A simplified schematic of the mobility-based frequency reference is shown in Fig. 2(a) [8]. It consists of a lowvoltage current mirror (formed by $M_{2,4}$ and $O A_{2}$ ) with gain $n=\frac{W_{4} / L_{4}}{W_{2} / L_{2}}$ and the NMOS pair $M_{1,3}$. The voltage difference between the gates of $M_{1}$ and $M_{3}$ is kept equal to $V_{R}$ by the the combination of the current source $I_{0}, R_{0}$ and $O A_{1}$. Using the square-law MOS model, the drain current of $M_{1}$ can be written as

$$
I_{1}=\frac{\mu_{n} C_{o x}}{2} \frac{W_{1}}{L_{1}} \frac{V_{R}^{2}}{\left(\sqrt{\frac{n}{m}}-1\right)^{2}}
$$

where $m=\frac{W_{3} / L_{3}}{W_{1} / L_{1}}, C_{o x}$ is the oxide capacitance per unit area and $\mu_{n}$ is the electron mobility [8]. The current source $I_{0}$ is implemented by mirroring the current flowing in a resistor matched to $R_{0}$ and whose voltage drop is equal to the reference voltage $V_{R}$ (not shown in the schematic).

The drain current of $M_{1}$ is mirrored by $M_{A}$ and $M_{B}$ with a gain of four and used to alternatively discharge $C_{A}$ and

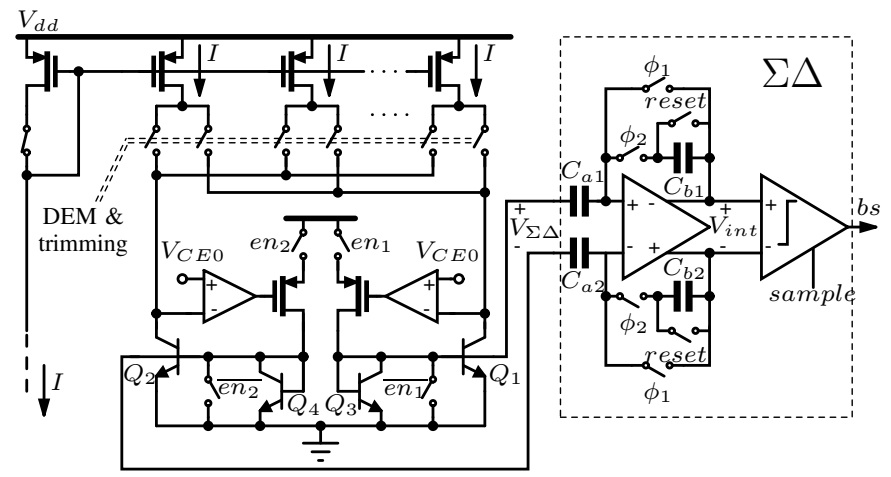

Fig. 3. Simplified schematic of the band-gap temperature sensor.

$C_{B}$ after they have been precharged to $V_{r 1}$. When the voltage on the discharging capacitor drops below $V_{r 2}$, the output of the comparator switches and the linear discharge of the other capacitor starts. The recharge is delayed by a short interval $D$, ensuring that the comparator's non-idealities do not affect the slope of the discharge at the $V_{r 2}$-crossing. Note that $D$ is not critical, as it does not influence the period $T$. Using (1), the oscillation frequency is

$$
f_{o s c}=\frac{\mu_{n} C_{o x}}{4 C\left(\sqrt{\frac{n}{m}}-1\right)^{2}} \frac{W_{1}}{L_{1}} \frac{V_{R}^{2}}{V_{r 1}-V_{r 2}}
$$

where $C=C_{A}=C_{B} \propto C_{o x}$. If $V_{R}, V_{r 1}$ and $V_{r 2}$, are reference voltages then $f_{\text {osc }}$ has the same temperature dependence as $\mu_{n}$.

The two multiplexers at the input of the comparator, driven by the signal chop shown in Fig. 2(b), are used to mitigate the effect of comparator offset.

\section{Temperature Sensor}

The band-gap based TS is shown in Fig. 3 [9]. When $e n_{1,2}$ are both high, the vertical NPN $Q_{1,2}$ are biased by the PMOS current sources array at a 1:4 collector current ratio to produce a PTAT difference between their base emitter voltages $V_{\Sigma \Delta}=\Delta V_{b e}$. When $e n_{1}\left(e n_{2}\right)$ is high and $e n_{2}\left(e n_{1}\right)$ is low, $Q_{1}\left(Q_{2}\right)$ is biased by a fixed current and the base-emitter junction of $Q_{2}\left(Q_{1}\right)$ is shorted to produce $V_{\Sigma \Delta}=+V_{b e}$ $\left(V_{\Sigma \Delta}=-V_{b e}\right)$. The feedback loops comprising the amplifiers and the common-source buffers compensate the base current of $Q_{1,2}$, so that neither $\Delta V_{b e}$ nor $V_{b e}$ depends on the bipolar current gain. Moreover, the two loops increase the output impedance at the the collectors of $Q_{1,2}$, by fixing the collector voltages equal to the reference voltage $V_{C E 0}$. To prevent the capacitive load of the analogue-to-digital converter from making the loops unstable, diode-connected $Q_{3,4}$ are added to lower the impedance at the base of $Q_{1,2}$.

A $1^{\text {st }}$-order $\Sigma \Delta$ analog-to-digital converter is used to produce an output bitstream $b s$ whose average $\mu$ represents the TS output. The switched-capacitor integrator in the $\Sigma \Delta$ integrates $2 \cdot \Delta V_{b e}$ when $b s=0$ and $-V_{b e}$ when $b s=1$. Since the negative feedback forces the average integrated voltage to be 


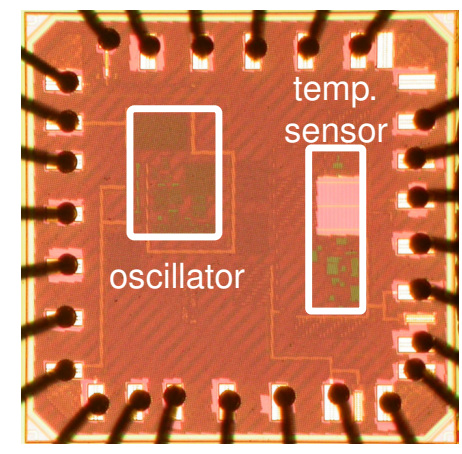

Fig. 4. Die micrograph of the test chip.

zero, the bitstream average is

$$
\mu=\langle b s\rangle=\frac{2 \cdot \Delta V_{b e}}{V_{b e}+2 \cdot \Delta V_{b e}}
$$

Although $\mu$ is a non-linear function of temperature, the biasing of the NPNs has been chosen [9] such that a function that is proportional-to-absolute-temperature (PTAT) can be obtained by applying the transformation

$$
\mu_{\text {PTAT }}(\mu)=\frac{9 \cdot \mu}{1+8 \cdot \mu}
$$

\section{Temperature compensation}

For flexibility, the temperature compensation scheme was implemented off-line in Matlab. However, it can be practically implemented without incurring much hardware complexity. For example, to determine fixed time intervals, the divide-by$N$ shown in Fig. 1 can be replaced by a simple counter. After an initial reset, the end of the time interval is denoted by the instant when the counter's output is equal to $M_{c y c} \propto N$, where $M_{c y c}$ can be adjusted in a temperature-dependent manner. For time intervals in the order of $T_{\text {meas }}=100 \mathrm{~ms}$ as required for WSN synchronization [1], $M_{\text {cyc }}=T_{\text {meas }} \cdot f_{\text {osc }} \approx 15 \cdot 10^{3}$ at room temperature, which is equivalent to more than 13 bits of temperature compensating resolution.

For a single-point trim, the oscillation frequency of each sample at the trim temperature $f_{\text {osc }}\left(T_{\text {trim }}\right)$ is measured. A seventh-order polynomial $P_{7}(\cdot)$, whose coefficients are fixed for all the samples, is then obtained via a batch calibration. The divider factor $N$ is computed as

$$
N=\frac{f_{\text {osc }}\left(T_{\text {trim }}\right)}{f_{\text {nom }}} P_{7}(\mu)
$$

where $f_{\text {nom }}=150 \mathrm{kHz}$ is the nominal frequency of oscillation, i.e. the desired output frequency.

For two-point trim, the following procedure is adopted. The oscillator frequency $f_{\text {osc }}$ and the on-chip TS decimated output $\mu$ are measured at two different temperatures, $T_{\text {trim,1 }}$ and $T_{\text {trim,2 }}$. Those data are used to interpolate the frequency using the interpolant

$$
f_{\text {osc }}=A \cdot \mu_{P T A T}^{B}
$$

where $\mu_{P T A T}$ is computed from the TS output using (4) and $A$ and $B$ are the trim parameters for each sample. A fourthorder polynomial $Q_{4}(\cdot)$ is obtained from batch calibration so

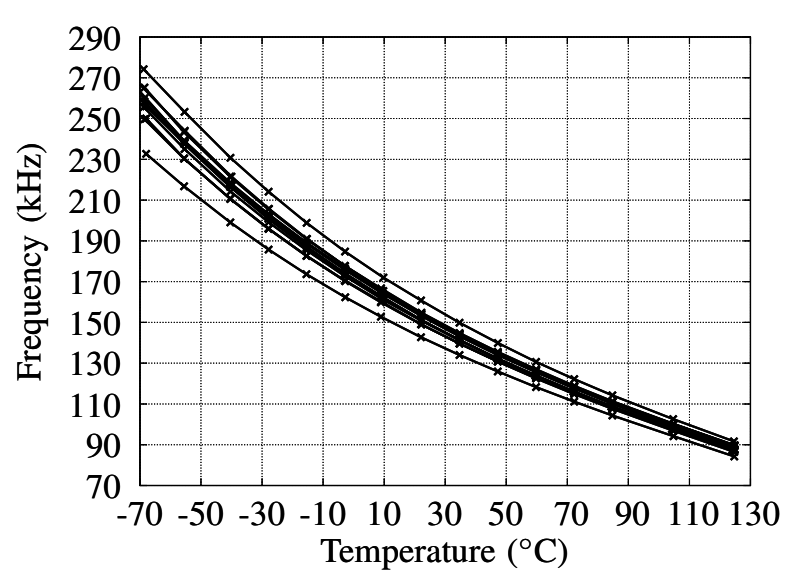

Fig. 5. Uncompensated oscillator output frequency $\left(f_{\text {osc }}\right)$.

that the divider factor $N$ computed for each sample is

$$
N=\frac{1}{f_{\text {nom }}} A \cdot\left\{\mu_{P T A T}\left[Q_{4}(\mu)\right]\right\}^{B}
$$

The polynomial ${ }^{1} Q_{4}(\cdot)$ is required to compensate for the fact that the power-law interpolant in (6) only approximately describes the temperature dependence of the electron mobility, especially over a wide temperature range.

\section{EXPERIMENTAL RESULTS}

The frequency reference was fabricated in a standard $65-\mathrm{nm}$ CMOS process (Fig. 4). The circuit occupies $0.2 \mathrm{~mm}^{2}(0.1$ $\mathrm{mm}^{2}$ for the oscillator and $0.1 \mathrm{~mm}^{2}$ for the TS) and uses only 2.5-V I/O thick oxide MOS devices. For flexibility, some control logic, the temperature sensor's $\operatorname{sinc}^{2}$ decimation filter and the reference voltages $V_{R}=0.25 \mathrm{~V}, V_{r 1}=1.6 \mathrm{~V}$ and $V_{r 2}=1.2 \mathrm{~V}$ were implemented off-chip. The reference draws $42.6 \mu \mathrm{A}(34.3 \mu \mathrm{A}$ for the oscillator and $8.3 \mu \mathrm{A}$ for the TS) from a 1.2-V supply at room temperature. The supply sensitivity is $1.2 \% / \mathrm{V}$.

Measurements on 12 samples from one batch were performed over the temperature range from $-70{ }^{\circ} \mathrm{C}$ to $+125{ }^{\circ} \mathrm{C}$ using a temperature-controlled oven. The temperature of the samples was measured using a Pt100 platinum thermometer and compared to the temperature reading of the on-chip TS. The TS shows a spread on $\mu_{P T A T}$ of $0.5^{\circ} \mathrm{C}(3 \sigma)$ over the range from $-70{ }^{\circ} \mathrm{C}$ to $+125{ }^{\circ} \mathrm{C}$.

Fig. 5 shows the uncompensated output frequency of the oscillator. At room temperature, its maximum deviation from the average is $\pm 6 \%$. First, the samples were trimmed at $T_{\text {trim }}=22{ }^{\circ} \mathrm{C}$ and compensated with an external Pt100 and an ideal temperature compensation curve. In those conditions, the maximum error is $\pm 2.6 \%$ over the military range from $-55{ }^{\circ} \mathrm{C}$ to $125{ }^{\circ} \mathrm{C}$. Then, the compensation polynomial $P_{7}(\cdot)$ (see section II-D) was extracted from batch calibration of the 12 devices. After a single-point trim at $T_{\text {trim }}=22{ }^{\circ} \mathrm{C}$, the

\footnotetext{
${ }^{1}$ Note that the order of the polynomials $P_{7}(\cdot)$ and $Q_{4}(\cdot)$ is the minimum required for the error due to the non-linearity of the compensation to be negligible compared to the spread among the samples.
} 


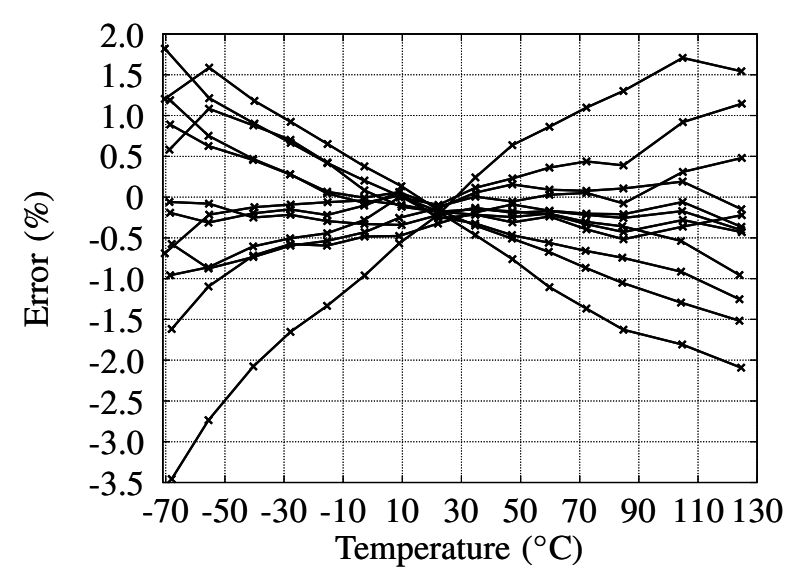

Fig. 6. Frequency error of the reference after single-point trim.

error when compensating with the on-chip TS is less than $\pm 2.7 \%$ (Fig. 6). Finally, a two-point trim at $T_{\text {trim }, 1}=-27{ }^{\circ} \mathrm{C}$ and $T_{\text {trim }, 2}=105{ }^{\circ} \mathrm{C}$ was employed and the error improved to $\pm 0.5 \%$ using another compensating polynomial $Q_{4}(\cdot)$ (see section II-D) extracted from a batch calibration of the 12 devices (Fig. 7). For the adopted compensation schemes, the resolution of the integer divider factor $N$ in Fig. 1 has been limited to 13 bits. Since this resolution can be easily reached in a practical implementation, as discussed in section II-D, the feasibility of the proposed compensation scheme is proved.

The frequency reference's performance is summarized in Table I and compared to other low-power fully integrated CMOS frequency reference. The proposed frequency reference achieves accuracy comparable to the state-of-the-art over a wider temperature range and for significantly more samples.

\section{CONCLUSIONS}

A fully integrated temperature-compensated frequency reference based on electron mobility has been presented. Its inaccuracy is less than $\pm 2.7 \%$ after single-point trim and less than $\pm 0.5 \%$ after two-point trim over the military temperature range. This demonstrates that frequency references with

TABLE I

PERformANCE SUMMARY AND COMPARISON.

\begin{tabular}{|c|c|c|c|c|c|}
\hline Reference & [6] & [7] & [10] & \multicolumn{2}{|c|}{ This work } \\
\hline Frequency & $6 \mathrm{MHz}$ & $10 \mathrm{MHz}$ & $30 \mathrm{MHz}$ & \multicolumn{2}{|c|}{$150 \mathrm{kHz}$} \\
\hline Supply & $1.2 \mathrm{~V}$ & $1.2 \mathrm{~V}$ & $3.3 \mathrm{~V}$ & \multicolumn{2}{|c|}{$1.2 \mathrm{~V}$} \\
\hline Power & $66 \mu \mathrm{W}$ & $80 \mu \mathrm{W}$ & $180 \mu \mathrm{W}$ & \multicolumn{2}{|c|}{$51 \mu \mathrm{W}$} \\
\hline Technology & $65 \mathrm{~nm}$ & $0.18 \mu \mathrm{m}$ & $0.35 \mu \mathrm{m}$ & \multicolumn{2}{|c|}{$65 \mathrm{~nm}$} \\
\hline Temp. range $\left({ }^{\circ} \mathrm{C}\right)$ & $0 \sim 120$ & $-20 \sim 100$ & $-20 \sim 100$ & \multicolumn{2}{|c|}{$-55 \sim 125$} \\
\hline Inaccuracy & $\pm 0.9 \%$ & $\pm 0.4 \%$ & $\pm 0.7 \%$ & $\pm 0.5 \%$ & $\pm 2.7 \%$ \\
\hline Calibration & single & N.A. $^{a}$ & N.A. ${ }^{a}$ & double & single \\
\hline $\begin{array}{l}\text { Samples tested } \\
\text { over temp. }\end{array}$ & 4 & 1 & 1 & \multicolumn{2}{|c|}{12} \\
\hline
\end{tabular}

${ }^{a}$ No calibration applied on a single sample.

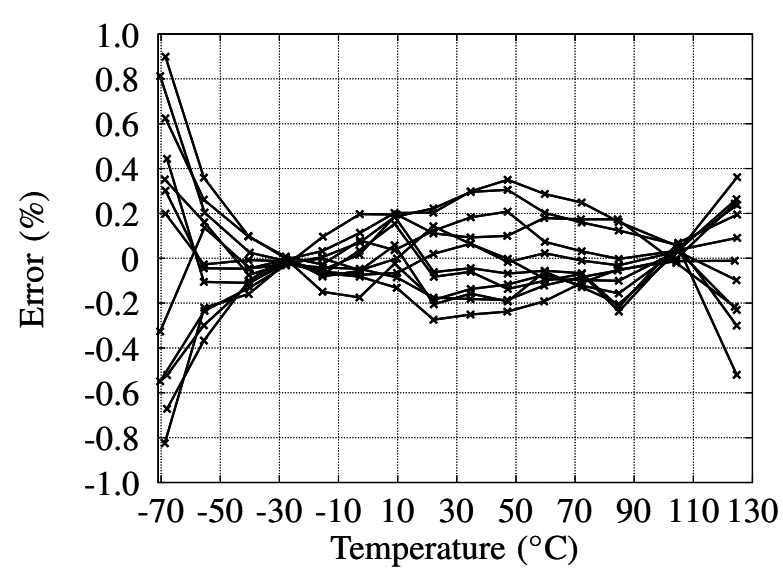

Fig. 7. Frequency error of the reference after two-point trim.

inaccuracies less than $1 \%$ over a wide temperature range can be realized with MOS transistors, even in nanometer CMOS. Those references are accurate enough for WSN applications, while working at low-voltage and low-power, as required for the use in autonomous sensor nodes.

\section{ACKNOWLEDGMENT}

This work is funded by the European Commission in the Marie Curie project TRANDSSAT - 2005-020461.

\section{REFERENCES}

[1] S. Drago, F. Sebastiano, L. Breems, D. Leenaerts, K. Makinwa, and B. Nauta, "Impulse based scheme for crystal-less ULP radios," IEEE Trans. Circuits Syst. I, pp. 1041 - 1052, May 2009.

[2] S. Drago, D. M. W. Leenaerts, F. Sebastiano, L. J. Breems, K. A. A. Makinwa, and B. Nauta, "A $2.4 \mathrm{GHz} 830 \mathrm{pJ} / \mathrm{bit}$ duty-cycled wake-up receiver with $-82 \mathrm{dbm}$ sensitivity for crystal-less wireless sensor nodes," in Proc. ISSCC, feb. 2010, pp. $224-225$.

[3] M. S. McCorquodale, S. M. Pernia, J. D. O’Day, G. Carichner, E. Marsman, N. Nguyen, S. Kubba, S. Nguyen, J. Kuhn, and R. B. Brown, "A 0.5 -to-480 MHz self-referenced CMOS clock generator with $90 \mathrm{ppm}$ total frequency error and spread-spectrum capability," in ISSCC Dig. of Tech. Papers, Feb. 2008, pp. 524 - 525.

[4] K. Sundaresan, P. Allen, and F. Ayazi, "Process and temperature compensation in a 7-MHz CMOS clock oscillator," IEEE J. Solid-State Circuits, vol. 41, no. 2, pp. 433-442, Feb. 2006.

[5] M. Kashmiri, M. Pertijs, and K. Makinwa, "A thermal-diffusivity-based frequency reference in standard CMOS with an absolute inaccuracy of $\pm 0.1 \%$ from $-55^{\circ} \mathrm{C}$ to $125^{\circ} \mathrm{C}$," in ISSCC Dig. Tech. Papers, Feb. 2010 , pp. $74-75,75 \mathrm{a}$.

[6] V. De Smedt, P. De Wit, W. Vereecken, and M. Steyaert, "A $66 \mu \mathrm{W}$ $86 \mathrm{ppm} /{ }^{\circ} \mathrm{C}$ fully-integrated $6 \mathrm{MHz}$ wienbridge oscillator with a $172 \mathrm{~dB}$ phase noise FOM,’ IEEE J. Solid-State Circuits, vol. 44, no. 7, pp. 1990 -2001, July 2009.

[7] J. Lee and S. Cho, "A $10 \mathrm{MHz} 80 \mu \mathrm{W} 67 \mathrm{ppm} /{ }^{\circ} \mathrm{C}$ CMOS reference clock oscillator with a temperature compensated feedback loop in 0.18 $\mu \mathrm{m}$ CMOS," in Proc. VLSI, June 2009, pp. $226-227$.

[8] F. Sebastiano, L. Breems, K. Makinwa, S. Drago, D. Leenaerts, and B. Nauta, "A low-voltage mobility-based frequency reference for crystalless ULP radios," IEEE J. Solid-State Circuits, vol. 44, no. 7, pp. 2002 -2009 , July 2009.

[9] — , "A $1.2 \mathrm{~V} 10 \mu \mathrm{W}$ NPN-based temperature sensor in $65 \mathrm{~nm}$ CMOS with an inaccuracy of $0.2^{\circ} \mathrm{C}(3 \sigma)$ from $-70^{\circ} \mathrm{C}$ to $125^{\circ} \mathrm{C}$," in ISSCC Dig. Tech. Papers, Feb. 2009, pp. 312 - 313, 313a.

[10] K. Ueno, T. Asai, and Y. Amemiya, "A $30 \mathrm{MHz}, 90-\mathrm{ppm} /{ }^{\circ} \mathrm{C}$ fullyintegrated clock reference generator with frequency-locked loop," in Proc. ESSCIRCs, Sept. 2009, pp. 392-395. 